\title{
RECURRENCE RELATIONS FOR TWO-CHANNEL CLOSED QUEUEING SYSTEMS WITH ERLANGIAN SERVICE TIMES
}

\author{
Bohdan Kopytko ${ }^{1}$, Kostiantyn Zhernovyi ${ }^{2}$ \\ ${ }^{I}$ Institute of Mathematics, Czestochowa University of Technology \\ Czestochowa, Poland \\ ${ }^{2}$ Educational and Scientific Institute, Banking University, Lviv, Ukraine \\ bohdan.kopytko@im.pcz.pl,k.zhernovyi@yahoo.com
}

Received: 12 December 2017; Accepted: 26 January 2018

\begin{abstract}
This paper proposes a method for determining the steady-state characteristics of two-channel closed queueing systems with an exponential distribution of the time generation of service requests and the Erlang distributions of the service times. Recurrence relations for computing the steady-state distribution of the number of customers in the system are deduced. The obtained algorithms are tested on examples using simulation models in the GPSS World environment.
\end{abstract}

MSC 2010: 60G10, 60J28, 60K25, $93 B 40$

Keywords: two-channel closed queueing system, Erlangian service times, fictitious phase method, recurrence relations

\section{Introduction}

Closed queueing systems are widely used as models to evaluate characteristics of the information systems, data networks and queueing processes in production, transport, trade, logistics and service systems [1]. The closed system is also called the system with a finite number of sources or the Engset system.

Suppose that a two-channel queueing system receives service requests from $m$ identical sources. Each source is alternately on and off. A source is off when it has a service request being served, otherwise the source is on. A source in the on-state generates a new service request after an exponentially distributed time (the generation time) with mean $1 / \lambda$. The sources act independently of each other. The service time of a service request has the Erlang distribution. A service request, that is generated when two channels are occupied, waits in the queue.

To investigate the systems with Erlangian service times, in particular the $M / E_{n} / 1 / \infty$ system [2], the method of fictitious phases, developed by A.K. Erlang [3], was applied. The Erlangian service times of the order $n$ means that each 
customer runs sequentially $n$ service phases, the duration of which is distributed exponentially with parameters $\mu_{1}, \mu_{2}, \ldots, \mu_{n}$ respectively.

The objective of this work is the construction with the help of fictitious phase method recursive algorithms for computing the steady-state distribution of the number of customers in two-channel closed queueing systems with an exponential distribution of the time generation of service requests and Erlangian service times of the order $n=2, n=3$ and $n \geq 4$. A similar approach is used in [4,5], where recursive algorithms are developed for the systems $M / E_{2} / 2 / m, M / E_{2} / 2 / \infty, M / E_{2} / 3 / m$ and $M / E_{2} / 3 / \infty$ as well as for the systems of the same types with threshold and hysteretic strategies of the random dropping of customers.

\section{The system with Erlangian service times of the second order}

Suppose that the service time of each customer is distributed under the generalized Erlang law of the order $n$, that is, the service time is the sum of $n$ independent random variables exponentially distributed with parameters $\mu_{1}, \mu_{2}, \ldots, \mu_{n}$ respectively.

Let $n_{c}$ denote the number of customers in the system and let $n_{b c}$ be the number of busy channels. In accordance with the method of phases, let us enumerate the system's states as follows: $s_{0}$ corresponds to the empty system; $s_{1\left(0_{j}\right)}$ is the state, when $n_{c}=n_{b c}=1$ and the service occurs in the phase $j(1 \leq j \leq n) ; s_{k(i j)}$ is the state, when $n_{c}=k(2 \leq k \leq m), n_{b c}=2$ and the services occur in the phase $i$ and $j(1 \leq i \leq n, i \leq j \leq n)$ respectively. We denote by $p_{0}, p_{1(0 j)}$ and $p_{k(i j)}$ respectively, steady-state probabilities that the system is in the each of these states. Assuming that $p_{1(0 j)}=p_{1(1 j)}(1 \leq j \leq n)$ and $f_{\lambda}(k)=(m-k) \lambda(0 \leq k \leq m-1)$, to calculate the steady-state probabilities, in the case of $n=2$ we obtain the system of equations:

$$
\begin{gathered}
-f_{\lambda}(0) p_{0}+\mu_{2} p_{1(02)}=0, \\
-\left(f_{\lambda}(1)+\mu_{2}\right) p_{1(02)}+\mu_{1} p_{1(01)}+2 \mu_{2} p_{2(22)}=0, \\
-\left(f_{\lambda}(2)+2 \mu_{2}\right) p_{2(22)}+\mu_{1} p_{2(12)}=0, \\
-\left(f_{\lambda}(1)+\mu_{1}\right) p_{1(01)}+m \lambda p_{0}+\mu_{2} p_{2(12)}=0 ; \\
-\left(f_{\lambda}(k)+2 \mu_{1}\right) p_{k(11)}+f_{\lambda}(k-1) p_{k-1(11)}+\mu_{2} p_{k+1(12)}=0, \\
2 \leq k \leq m-1 ; \\
-\left(f_{\lambda}(k)+\mu_{1}+\mu_{2}\right) p_{k(12)}+f_{\lambda}(k-1) p_{k-1(12)}+2 \mu_{1} p_{k(11)}+2 \mu_{2} p_{k+1(22)}=0, \\
-\left(f_{\lambda}(k)+2 \mu_{2}\right) p_{k(22)}+f_{\lambda}(k-1) p_{k-1(22)}+\mu_{1} p_{k(12)}=0, \\
3 \leq k \leq m-1 ;
\end{gathered}
$$




$$
\begin{aligned}
& -2 \mu_{1} p_{m(11)}+f_{\lambda}(m-1) p_{m-1(11)}=0, \\
& -\left(\mu_{1}+\mu_{2}\right) p_{m(12)}+f_{\lambda}(m-1) p_{m-1(12)}+2 \mu_{1} p_{m(11)}=0, \\
& -2 \mu_{2} p_{m(22)}+f_{\lambda}(m-1) p_{m-1(22)}+\mu_{1} p_{m(12)}=0 ; \\
& p_{0}+p_{1(01)}+p_{1(02)}+\sum_{k=2}^{m}\left(p_{k(11)}+p_{k(12)}+p_{k(22)}\right)=1 .
\end{aligned}
$$

Introducing the notation

$$
\begin{aligned}
& \alpha_{i}=\frac{\lambda}{\mu_{i}}, 1 \leq i \leq n ; \quad \eta_{i}=\frac{\mu_{i}}{\mu_{1}}, 1 \leq i \leq n ; \quad g_{\alpha}(k)=(m-k) \alpha_{1}, \quad 0 \leq k \leq m-1 ; \\
& \tilde{p}_{k(i j)}=\frac{p_{k(i j)}}{p_{0}}, 1 \leq k \leq m, 1 \leq i \leq n, \quad i \leq j \leq n,
\end{aligned}
$$

and using equations (1), we find:

$$
\begin{aligned}
& \tilde{p}_{1(02)}=m \alpha_{2}, \quad \tilde{p}_{2(22)}=\frac{m\left(\alpha_{2}\left(g_{\alpha}(1)+1\right)\left(g_{\alpha}(1)+\eta_{2}\right)-\alpha_{1}\right)}{\eta_{2}\left((3 m-4) \alpha_{1}+\eta_{2}+2\right)}, \\
& \tilde{p}_{1(01)}=m \alpha_{2}\left(g_{\alpha}(1)+\eta_{2}\right)-2 \eta_{2} \tilde{p}_{2(22)}, \quad \tilde{p}_{2(12)}=\left(g_{\alpha}(2)+\eta_{2}\right) \tilde{p}_{2(22)} .
\end{aligned}
$$

From equations (2), we obtain the recurrence relations:

$$
\begin{aligned}
& \tilde{p}_{k(22)}=f_{22}\left(\tilde{p}_{k-1(22)}, \tilde{p}_{k-2(11)}, \tilde{p}_{k-2(12)}, \tilde{p}_{k-1(12)}\right), \quad 3 \leq k \leq m-1 ; \\
& \tilde{p}_{k(12)}=f_{12}\left(\tilde{p}_{k 22}, \tilde{p}_{k-1,22}\right), \quad 3 \leq k \leq m-1 ; \\
& \tilde{p}_{k(11)}=f_{11}\left(\tilde{p}_{k(12)}, \tilde{p}_{k-1(12)}, \tilde{p}_{k+1(22)}\right), \quad 2 \leq k \leq m-1,
\end{aligned}
$$

where:

$$
\begin{aligned}
& f_{22}(x, y, z, u)=\frac{g(x, y, z, u)}{2 \eta_{2}\left(g_{\alpha}(k)+2 \eta_{2}+g_{\alpha}(k-1)+2\right)}, \\
& g(x, y, z, u)=2 \eta_{2} g_{\alpha}(k-1) x-2 g_{\alpha}(k-2) y- \\
& \quad-\left(g_{\alpha}(k-1)+2\right)\left(g_{\alpha}(k-2) z-\left(g_{\alpha}(k-1)+\eta_{2}+1\right) u\right) ; \\
& f_{12}(x, y)=\left(g_{\alpha}(k)+2 \eta_{2}\right) x-g_{\alpha}(k-1) y ; \\
& f_{11}(x, y, z)=\frac{g_{\alpha}(k)+\eta_{2}+1}{2} x-\frac{g_{\alpha}(k-1)}{2} y-\eta_{2} z .
\end{aligned}
$$

Using equations (3) we find: 


$$
\begin{aligned}
& \tilde{p}_{m(12)}=\frac{\alpha_{1}}{\alpha_{1}+2 \eta_{2}+2}\left(\left(\alpha_{1}+\eta_{2}+3\right) \tilde{p}_{m-1(12)}-\alpha_{1}\left(\tilde{p}_{m-1(22)}+2 \tilde{p}_{m-2(12)}\right)\right), \\
& \tilde{p}_{m(22)}=\frac{1}{2 \eta_{2}}\left(\alpha_{1} \tilde{p}_{m-1(22)}+\tilde{p}_{m(12)}\right), \quad \tilde{p}_{m(11)}=\frac{\alpha_{1}}{2} \tilde{p}_{m-1(11)} .
\end{aligned}
$$

Recurrence relations (5)-(7) allow us to consistently calculate $\tilde{p}_{1(02)}, \tilde{p}_{2(22)}$, $\tilde{p}_{1(01)}, \tilde{p}_{2(12)} ; \tilde{p}_{k(22)}, \tilde{p}_{k(12)}, \tilde{p}_{k-1(11)}(3 \leq k \leq m-1), \tilde{p}_{m(12)}, \tilde{p}_{m(22)}, \tilde{p}_{m-1(11)}$ and $\tilde{p}_{m(11)}$. Using the normalization condition (4), we find steady-state probabilities by the formulas

$$
\begin{aligned}
& p_{0}=\left(1+\sum_{j=1}^{n} \tilde{p}_{1(0 j)}+\sum_{k=2}^{m} \sum_{i=1}^{n} \sum_{j=i}^{n} \tilde{p}_{k(i j)}\right)^{-1} ; \quad p_{k}=p_{0} \tilde{p}_{k}, \quad 1 \leq k \leq m ; \\
& \tilde{p}_{1}=\sum_{j=1}^{n} \tilde{p}_{1(0 j)} ; \quad \tilde{p}_{k}=\sum_{i=1}^{n} \sum_{j=i}^{n} \tilde{p}_{k(i j)}, \quad 2 \leq k \leq m .
\end{aligned}
$$

Here $n=2$ and $p_{k}$ is the steady-state probability that $n_{c}=k$. We calculate the steady-state characteristics - the average number of customers in the system $\mathbf{E}\left(n_{c}\right)$, the average queue length $\mathbf{E}(Q)$ and average waiting time $\mathbf{E}(W)$ - by the formulas

$$
\mathbf{E}\left(n_{c}\right)=\sum_{k=1}^{m} k p_{k}, \quad \mathbf{E}(Q)=\sum_{k=1}^{m-2} k p_{k+2}, \quad \mathbf{E}(W)=\frac{\mathbf{E}(Q)}{\lambda_{\mathrm{av}}} .
$$

Here $\lambda_{\mathrm{av}}$ is a steady-state value of the arrival rate of customers, defined by the equality

$$
\lambda_{\mathrm{av}}=\lambda \sum_{k=0}^{m-1}(m-k) p_{k}
$$

The parameter $\lambda_{\mathrm{av}}$ is a characteristic of the system capacity, because for the steady-state regime we have the equality of the intensities of flows of customers arriving and served.

\section{The system with Erlangian service times of the third order}

To calculate the steady-state probabilities, in the case of $n=3$ we obtain the system of equations: 


$$
\begin{aligned}
& -f_{\lambda}(0) p_{0}+\mu_{3} p_{1(03)}=0 \\
& -\left(f_{\lambda}(1)+\mu_{1}\right) p_{1(01)}+f_{\lambda}(0) p_{0}+\mu_{3} p_{2(13)}=0 \text {; } \\
& -\left(f_{\lambda}(1)+\mu_{2}\right) p_{1(02)}+\mu_{3} p_{2(23)}+\mu_{1} p_{1(01)}=0 \text {; } \\
& -\left(f_{\lambda}(1)+\mu_{3}\right) p_{1(03)}+2 \mu_{3} p_{2(33)}+\mu_{2} p_{1(02)}=0 \text {; } \\
& -\left(f_{\lambda}(k)+2 \mu_{1}\right) p_{k(11)}+f_{\lambda}(k-1) p_{k-1(11)}+\mu_{3} p_{k+1(13)}=0, \quad 2 \leq k \leq m-1 \text {; } \\
& -2 \mu_{1} p_{m(11)}+f_{\lambda}(m-1) p_{m-1(11)}=0 \text {; } \\
& -\left(f_{\lambda}(k)+\mu_{1}+\mu_{2}\right) p_{k(12)}+f_{\lambda}(k-1) p_{k-1(12)}+\mu_{3} p_{k+1(23)}+2 \mu_{1} p_{k(11)}=0 \text {, } \\
& 2 \leq k \leq m-1 \\
& -\left(\mu_{1}+\mu_{2}\right) p_{m(12)}+f_{\lambda}(m-1) p_{m-1(12)}+2 \mu_{1} p_{m(11)}=0 ; \\
& -\left(f_{\lambda}(k)+\mu_{1}+\mu_{3}\right) p_{k(13)}+f_{\lambda}(k-1) p_{k-1(13)}+2 \mu_{3} p_{k+1(33)}+\mu_{2} p_{k(12)}=0 \text {, } \\
& 2 \leq k \leq m-1 \\
& -\left(\mu_{1}+\mu_{3}\right) p_{m(13)}+f_{\lambda}(m-1) p_{m-1(13)}+\mu_{2} p_{m(12)}=0 \text {; } \\
& -\left(f_{\lambda}(2)+2 \mu_{i}\right) p_{2(i i)}+\mu_{i-1} p_{2(i-1, i)}=0, \quad i=2,3 \text {; } \\
& -\left(f_{\lambda}(2)+\mu_{2}+\mu_{3}\right) p_{2(23)}+2 \mu_{2} p_{2(22)}+\mu_{1} p_{2(13)}=0 \text {; } \\
& -\left(f_{\lambda}(k)+2 \mu_{i}\right) p_{k(i i)}+f_{\lambda}(k-1) p_{k-1(i i)}+\mu_{i-1} p_{k(i-1, i)}=0 \text {, } \\
& 3 \leq k \leq m-1, \quad i=2,3 \\
& -2 \mu_{i} p_{m(i i)}+f_{\lambda}(m-1) p_{m-1(i i)}+\mu_{i-1} p_{m(i-1, i)}=0, \quad i=2,3 \text {; } \\
& -\left(f_{\lambda}(k)+\mu_{2}+\mu_{3}\right) p_{k(23)}+f_{\lambda}(k-1) p_{k-1(23)}+2 \mu_{2} p_{k(22)}+\mu_{1} p_{k(13)}=0 \text {, } \\
& 3 \leq k \leq m-1 \\
& -\left(\mu_{2}+\mu_{3}\right) p_{m(23)}+f_{\lambda}(m-1) p_{m-1(23)}+2 \mu_{2} p_{m(22)}+\mu_{1} p_{m(13)}=0 \text {; } \\
& p_{0}+\sum_{j=1}^{3} p_{1(0 j)}+\sum_{k=2}^{m} \sum_{i=1}^{3} \sum_{j=i}^{3} p_{k(i j)}=1
\end{aligned}
$$

Introducing the notation $\tilde{p}_{2(33)}=p$ and using equations (9) we find:

$$
\begin{aligned}
& \tilde{p}_{1(03)}=m \alpha_{3}, \quad \tilde{p}_{1(02)}=\frac{1}{\eta_{2}}\left(\left(g_{\alpha}(1)+\eta_{3}\right) \tilde{p}_{1(03)}-2 \eta_{3} p\right), \quad \tilde{p}_{2(23)}=\frac{g_{\alpha}(2)+2 \eta_{3}}{\eta_{2}} p ; \\
& \tilde{p}_{1(01)}=\left(g_{\alpha}(1)+\eta_{2}\right) \tilde{p}_{1(02)}-\eta_{3} \tilde{p}_{2(23)}, \quad \tilde{p}_{2(13)}=\frac{1}{\eta_{3}}\left(\left(g_{\alpha}(1)+1\right) \tilde{p}_{1(01)}-\alpha_{1}\right) ; \\
& \tilde{p}_{2(22)}=\frac{1}{2 \eta_{2}}\left(\left(g_{\alpha}(2)+\eta_{2}+\eta_{3}\right) \tilde{p}_{2(23)}-\tilde{p}_{2(13)}\right), \quad \tilde{p}_{2(12)}=\left(g_{\alpha}(2)+2 \eta_{2}\right) \tilde{p}_{2(22)} ;
\end{aligned}
$$




$$
\begin{aligned}
& \tilde{p}_{k(33)}=\frac{1}{2 \eta_{3}}\left(\left(1+g_{\alpha}(k-1)+\eta_{3}\right) \tilde{p}_{k-1(13)}-g_{\alpha}(k-2) \tilde{p}_{k-2(13)}-\eta_{2} \tilde{p}_{k-1(12)}\right), \quad 3 \leq k \leq m ; \\
& \tilde{p}_{k(23)}=\frac{1}{\eta_{2}}\left(\left(g_{\alpha}(k)+2 \eta_{3}\right) \tilde{p}_{k(33)}-g_{\alpha}(k-1) \tilde{p}_{k-1(33)}\right), \quad 3 \leq k \leq m-1 ; \\
& \tilde{p}_{m(23)}=\frac{1}{\eta_{2}}\left(2 \eta_{3} \tilde{p}_{m(33)}-g_{\alpha}(m-1) \tilde{p}_{m-1(33)}\right) ; \\
& \tilde{p}_{k(11)}=\frac{1}{2}\left(\left(1+g_{\alpha}(k)+\eta_{2}\right) \tilde{p}_{k(12)}-g_{\alpha}(k-1) \tilde{p}_{k-1(12)}-\eta_{3} \tilde{p}_{k+1(23)}\right), \quad 2 \leq k \leq m-1 ; \\
& \tilde{p}_{m(11)}=\frac{g_{\alpha}(m-1)}{2} \tilde{p}_{m-1(11)} ; \\
& \tilde{p}_{k(13)}=\frac{1}{\eta_{3}}\left(\left(g_{\alpha}(k-1)+2\right) \tilde{p}_{k-1(11)}-g_{\alpha}(k-2) \tilde{p}_{k-2(11)}\right), \quad 3 \leq k \leq m ; \\
& \tilde{p}_{k(22)}=\frac{1}{2 \eta_{2}}\left(\left(\eta_{2}+g_{\alpha}(k)+\eta_{3}\right) \tilde{p}_{k(23)}-g_{\alpha}(k-1) \tilde{p}_{k-1(23)}-\tilde{p}_{k(13)}\right), \\
& \tilde{p}_{m(22)}=\frac{1}{2 \eta_{2}}\left(\left(\eta_{2}+\eta_{3}\right) \tilde{p}_{m(23)}-g_{\alpha}(m-1) \tilde{p}_{m-1(23)}-\tilde{p}_{m(13)}\right) ; \\
& \left.\tilde{p}_{k(12)}=\left(2 \eta_{2}+g_{\alpha}(k)\right) \tilde{p}_{k(22)}-g_{\alpha}(k-1)\right) \tilde{p}_{k-1(22)}, \quad 3 \leq k \leq m-1 ; \\
& \left.\tilde{p}_{m(12)}=2 \eta_{2} \tilde{p}_{m(22)}-g_{\alpha}(m-1)\right) \tilde{p}_{m-1(22)} .
\end{aligned}
$$

Recurrence relations (11) allow us to calculate $\tilde{p}_{1(03)}$ and consistently obtain the expression for $\tilde{p}_{1(03)}, \tilde{p}_{1(02)}, \tilde{p}_{2(23)}, \tilde{p}_{1(01)}, \tilde{p}_{2(13)}, \tilde{p}_{2(22)}, \tilde{p}_{2(12)} ; \tilde{p}_{k(33)}, \tilde{p}_{k(23)}$, $\tilde{p}_{k-1(11)}, \tilde{p}_{k(13)}, \tilde{p}_{k(22)}, \tilde{p}_{k(12)}, 3 \leq k \leq m$, and $\tilde{p}_{m(11)}$ as linear functions of the unknown parameter $p=\tilde{p}_{2(33)}$. To find $p$, we use any of the equations

$$
\begin{aligned}
& -\left(\mu_{1}+\mu_{2}\right) p_{m(12)}+f_{\lambda}(m-1) p_{m-1(12)}+2 \mu_{1} p_{m(11)}=0 \\
& -\left(\mu_{1}+\mu_{3}\right) p_{m(13)}+f_{\lambda}(m-1) p_{m-1(13)}+\mu_{2} p_{m(12)}=0 .
\end{aligned}
$$

Equations (12) were not involved in obtaining (11). Using the normalization condition (10), we find the steady-state probabilities by (8).

\section{The system with Erlangian service times of $\boldsymbol{n}$-th order}

To calculate the steady-state probabilities in the case of $n \geq 4$, we obtain the system of equations: 
$-f_{\lambda}(0) p_{0}+\mu_{n} p_{1(0 n)}=0$

$-\left(f_{\lambda}(1)+\mu_{1}\right) p_{1(01)}+f_{\lambda}(0) p_{0}+\mu_{n} p_{2(1 n)}=0$;

$-\left(f_{\lambda}(1)+\mu_{j}\right) p_{1(0 j)}+\mu_{n} p_{2(j n)}+\mu_{j-1} p_{1(0, j-1)}=0,2 \leq j \leq n-1 ;$

$-\left(f_{\lambda}(1)+\mu_{n}\right) p_{1(0 n)}+2 \mu_{n} p_{2(n n)}+\mu_{n-1} p_{1(0, n-1)}=0$;

$-\left(f_{\lambda}(k)+2 \mu_{1}\right) p_{k(11)}+f_{\lambda}(k-1) p_{k-1(11)}+\mu_{n} p_{k+1(1 n)}=0, \quad 2 \leq k \leq m-1$;

$-2 \mu_{1} p_{m(11)}+f_{\lambda}(m-1) p_{m-1(11)}=0$;

$-\left(f_{\lambda}(k)+\mu_{1}+\mu_{2}\right) p_{k(12)}+f_{\lambda}(k-1) p_{k-1(12)}+\mu_{n} p_{k+1(2 n)}+2 \mu_{1} p_{k(11)}=0$,

$2 \leq k \leq m-1$

$-\left(\mu_{1}+\mu_{2}\right) p_{m(12)}+f_{\lambda}(m-1) p_{m-1(12)}+2 \mu_{1} p_{m(11)}=0 ;$

$-\left(f_{\lambda}(k)+\mu_{1}+\mu_{j}\right) p_{k(1 j)}+f_{\lambda}(k-1) p_{k-1(1 j)}+\mu_{n} p_{k+1(j n)}+\mu_{j-1} p_{k(1, j-1)}=0$, $2 \leq k \leq m-1,3 \leq j \leq n-1 ;$

$-\left(\mu_{1}+\mu_{j}\right) p_{m(1 j)}+f_{\lambda}(m-1) p_{m-1(1 j)}+\mu_{j-1} p_{m(1, j-1)}=0, \quad 3 \leq j \leq n$;

$-\left(f_{\lambda}(k)+\mu_{1}+\mu_{n}\right) p_{k(1 n)}+f_{\lambda}(k-1) p_{k-1(1 n)}+2 \mu_{n} p_{k+1(n n)}+\mu_{n-1} p_{k(1, n-1)}=0$, $2 \leq k \leq m-1$

$-\left(\mu_{1}+\mu_{n}\right) p_{m(1 n)}+f_{\lambda}(m-1) p_{m-1(1 n)}+\mu_{n-1} p_{m(1, n-1)}=0 ;$

$-\left(\lambda+2 \mu_{i}\right) p_{2(i i)}+\mu_{i-1} p_{2(i-1, i)}=0, \quad 2 \leq i \leq n ;$

$-\left(\lambda+\mu_{i}+\mu_{i+1}\right) p_{2(i, i+1)}+2 \mu_{i} p_{2(i i)}+\mu_{i-1} p_{2(i-1, i+1)}=0, \quad 2 \leq i \leq n-1 ;$

$-\left(\lambda+\mu_{i}+\mu_{j}\right) p_{2(i j)}+\mu_{i-1} p_{2(i-1, j)}+\mu_{j-1} p_{2(i, j-1)}=0, \quad 2 \leq i \leq n-2$,

$i+2 \leq j \leq n$

$-\left(f_{\lambda}(k)+2 \mu_{i}\right) p_{k(i i)}+f_{\lambda}(k-1) p_{k-1(i i)}+\mu_{i-1} p_{k(i-1, i)}=0, \quad 3 \leq k \leq m-1$,

$2 \leq i \leq n$

$-2 \mu_{i} p_{m(i i)}+f_{\lambda}(m-1) p_{m-1(i i)}+\mu_{i-1} p_{m(i-1, i)}=0,2 \leq i \leq n ;$

$-\left(f_{\lambda}(k)+\mu_{i}+\mu_{i+1}\right) p_{k(i, i+1)}+f_{\lambda}(k-1) p_{k-1(i, i+1)}+2 \mu_{i} p_{k(i i)}+\mu_{i-1} p_{k(i-1, i+1)}=0$,

$3 \leq k \leq m-1,2 \leq i \leq n-1$;

$-\left(\mu_{i}+\mu_{i+1}\right) p_{m(i, i+1)}+f_{\lambda}(m-1) p_{m-1(i, i+1)}+2 \mu_{i} p_{m(i i)}+\mu_{i-1} p_{m(i-1, i+1)}=0$,

$2 \leq i \leq n-1$

$-\left(f_{\lambda}(k)+\mu_{i}+\mu_{j}\right) p_{k(i j)}+f_{\lambda}(k-1) p_{k-1(i j)}+\mu_{i-1} p_{k(i-1, j)}+\mu_{j-1} p_{k(i, j-1)}=0$,

$3 \leq k \leq m-1,2 \leq i \leq n-2, i+2 \leq j \leq n ;$

$-\left(\mu_{i}+\mu_{j}\right) p_{m(i j)}+f_{\lambda}(m-1) p_{m-1(i j)}+\mu_{i-1} p_{m(i-1, j)}+\mu_{j-1} p_{m(i, j-1)}=0$,

$2 \leq i \leq n-2, i+2 \leq j \leq n$ 


$$
p_{0}+\sum_{j=1}^{n} p_{1(0 j)}+\sum_{k=2}^{m} \sum_{i=1}^{n} \sum_{j=i}^{n} p_{k(i j)}=1 .
$$

Introducing the notation $\tilde{p}_{k(i n)}=p_{k i}, 2 \leq k \leq m, 1 \leq i \leq n$, and using equations (13) we find:

$$
\begin{aligned}
& \tilde{p}_{1(0 n)}=m \alpha_{n}, \quad \tilde{p}_{1(01)}=\frac{1}{g_{\alpha}(1)+1}\left(g_{\alpha}(0)+\eta_{n} p_{21}\right), \\
& \tilde{p}_{1(0 j)}=\frac{1}{g_{\alpha}(1)+\eta_{j}}\left(\eta_{j-1} \tilde{p}_{1(0, j-1)}+\eta_{n} p_{2 j}\right), \quad 2 \leq j \leq n-1 ; \\
& \tilde{p}_{k(11)}=\frac{1}{g_{\alpha}(k)+2}\left(g_{\alpha}(k-1) \tilde{p}_{k-1(11)}+\eta_{n} p_{k+1,1}\right), \quad 2 \leq k \leq m-1 \text {; } \\
& \tilde{p}_{m(11)}=\frac{g_{\alpha}(m-1)}{2} \tilde{p}_{m-1(11)} \text {; } \\
& \tilde{p}_{k(12)}=\frac{1}{g_{\alpha}(k)+\eta_{2}+1}\left(g_{\alpha}(k-1) \tilde{p}_{k-1(12)}+2 \tilde{p}_{k(11)}+\eta_{n} p_{k+1,2}\right), \\
& 2 \leq k \leq m-1 ; \\
& \tilde{p}_{m(12)}=\frac{1}{\eta_{2}+1}\left(g_{\alpha}(m-1) \tilde{p}_{m-1(12)}+2 \tilde{p}_{m(11)}\right) ; \\
& \tilde{p}_{k(1 j)}=\frac{1}{g_{\alpha}(k)+\eta_{j}+1}\left(g_{\alpha}(k-1) \tilde{p}_{k-1(1 j)}+\eta_{j-1} \tilde{p}_{k(1, j-1)}+\eta_{n} p_{k+1, j}\right), \\
& 2 \leq k \leq m-1,3 \leq j \leq n-1 ; \\
& \tilde{p}_{m(1 j)}=\frac{1}{\eta_{j}+1}\left(g_{\alpha}(m-1) \tilde{p}_{m-1(1 j)}+\eta_{j-1} \tilde{p}_{m(1, j-1)}\right), 3 \leq j \leq n-1 ; \\
& \tilde{p}_{2(i i)}=\frac{\eta_{i-1}}{g_{\alpha}(2)+2 \eta_{i}} \tilde{p}_{2(i-1, i)}, \quad 2 \leq i \leq n-1 \\
& \tilde{p}_{k(i i)}=\frac{1}{g_{\alpha}(k)+2 \eta_{i}}\left(g_{\alpha}(k-1) \tilde{p}_{k-1(i i)}+\eta_{i-1} \tilde{p}_{k(i-1, i)}\right), \\
& 3 \leq k \leq m-1,2 \leq i \leq n-1 ; \\
& \tilde{p}_{m(i i)}=\frac{1}{2 \eta_{i}}\left(g_{\alpha}(m-1) \tilde{p}_{m-1(i i)}+\eta_{i-1} \tilde{p}_{m(i-1, i)}\right), 2 \leq i \leq n-1 ; \\
& \tilde{p}_{2(i, i+1)}=\frac{1}{g_{\alpha}(2)+\eta_{i}+\eta_{i+1}}\left(2 \eta_{i} \tilde{p}_{2(i i)}+\eta_{i-1} \tilde{p}_{2(i-1, i+1)}\right), \quad 2 \leq i \leq n-2
\end{aligned}
$$




$$
\begin{aligned}
& \tilde{p}_{2(i j)}=\frac{1}{g_{\alpha}(2)+\eta_{i}+\eta_{j}}\left(\eta_{i-1} \tilde{p}_{2(i-1, j)}+\eta_{j-1} \tilde{p}_{2(i, j-1)}\right), \\
& 2 \leq i \leq n-3, i+2 \leq j \leq n-1 ; \\
& \tilde{p}_{k(i, i+1)}=\frac{1}{g_{\alpha}(k)+\eta_{i}+\eta_{i+1}}\left(g_{\alpha}(k-1) \tilde{p}_{k-1(i, i+1)}+2 \eta_{i} \tilde{p}_{k(i i)}+\eta_{i-1} \tilde{p}_{k(i-1, i+1)}\right), \\
& \quad 3 \leq k \leq m-1,2 \leq j \leq n-2 ; \\
& \tilde{p}_{m(i, i+1)}=\frac{1}{\eta_{i}+\eta_{i+1}}\left(g_{\alpha}(m-1) \tilde{p}_{m-1(i, i+1)}+2 \eta_{i} \tilde{p}_{m(i i)}+\eta_{i-1} \tilde{p}_{m(i-1, i+1)}\right), \\
& \tilde{p}_{k(i j)}=\frac{1}{g_{\alpha}(k)+\eta_{i}+\eta_{j}}\left(g_{\alpha}(k-1) \tilde{p}_{k-1(i j)}+\eta_{i-1} \tilde{p}_{k(i-1, j)}+\eta_{j-1} \tilde{p}_{k(i, j-1)}\right), \\
& 3 \leq k \leq m-1,2 \leq i \leq n-3, i+2 \leq j \leq n-1 ; \\
& \tilde{p}_{m(i j)}=\frac{1}{\eta_{i}+\eta_{j}}\left(g_{\alpha}(m-1) \tilde{p}_{m-1(i j)}+\eta_{i-1} \tilde{p}_{m(i-1, j)}+\eta_{j-1} \tilde{p}_{m(i, j-1)}\right), \\
& 2 \leq i \leq n-3, i+2 \leq j \leq n-1 .
\end{aligned}
$$

Recurrence relations (15) allow us to calculate $\tilde{p}_{1(0 n)}$ and consistently obtain the expression for

$$
\begin{aligned}
& \tilde{p}_{1(0 n)} ; \quad \tilde{p}_{1(0 j)}, 1 \leq j \leq n-1 ; \quad \tilde{p}_{k(11)}, 2 \leq k \leq m ; \quad \tilde{p}_{k(12)}, 2 \leq k \leq m ; \\
& \tilde{p}_{k(13)}, \tilde{p}_{k(14)}, \ldots, \tilde{p}_{k(1, n-1)}, 2 \leq k \leq m-1 ; \quad \tilde{p}_{m(1 j)}, 3 \leq j \leq n-1 ; \\
& \tilde{p}_{k(22)}, 2 \leq k \leq m ; \quad \tilde{p}_{2(2 j)}, 3 \leq j \leq n-1 ; \quad \tilde{p}_{k(23)}, 3 \leq k \leq m ; \\
& \tilde{p}_{k(24)}, \tilde{p}_{k(25)}, \ldots, \tilde{p}_{k(2, n-1)}, 3 \leq k \leq m-1 ; \quad \tilde{p}_{m(2 j)}, 4 \leq j \leq n-1 ; \\
& \tilde{p}_{k(33)}, 2 \leq k \leq m ; \quad \tilde{p}_{2(3 j)}, 4 \leq j \leq n-1 ; \quad \tilde{p}_{k(34)}, 3 \leq k \leq m ; \\
& \tilde{p}_{k(35)}, \tilde{p}_{k(36)}, \ldots, \tilde{p}_{k(3, n-1)}, 3 \leq k \leq m-1 ; \quad \tilde{p}_{m(3 j)}, 5 \leq j \leq n-1 ; \\
& \tilde{p}_{k(i i)}, 2 \leq k \leq m ; \quad \tilde{p}_{2(i j)}, i+1 \leq j \leq n-1 ; \quad \tilde{p}_{k(i, i+1)}, 3 \leq k \leq m ; \\
& \tilde{p}_{k(i, i+2)}, \tilde{p}_{k(i, i+3)}, \ldots, \tilde{p}_{k(i, n-1)}, 3 \leq k \leq m-1 ; \quad \tilde{p}_{m(i j)}, i+2 \leq j \leq n-1 ; \\
& \tilde{p}_{k(n-2, n-2)}, 2 \leq k \leq m ; \quad \tilde{p}_{k(n-2, n-1)}, 3 \leq k \leq m ; \\
& \tilde{p}_{k(n-1, n-1)}, 2 \leq k \leq m,
\end{aligned}
$$

as linear functions of the unknown parameters $p_{k i}(2 \leq k \leq m, 1 \leq i \leq n)$. To determine $p_{k i}$, we use system of $n(m+1)$ equations 


$$
\begin{aligned}
& -\left(f_{\lambda}(k)+\mu_{1}+\mu_{n}\right) p_{k(1 n)}+f_{\lambda}(k-1) p_{k-1(1 n)}+2 \mu_{n} p_{k+1(n n)}+ \\
& \quad+\mu_{n-1} p_{k(1, n-1)}=0, \quad 2 \leq k \leq m-1 ; \\
& -\left(\mu_{1}+\mu_{n}\right) p_{m(1 n)}+f_{\lambda}(m-1) p_{m-1(1 n)}+\mu_{n-1} p_{m(1, n-1)}=0 ; \\
& -\left(f_{\lambda}(2)+2 \mu_{n}\right) p_{2(n n)}+\mu_{n-1} p_{2(n-1, n)}=0 ; \\
& -\left(f_{\lambda}(2)+\mu_{n-1}+\mu_{n}\right) p_{2(n-1 n s)}+2 \mu_{n-1} p_{2(n-1, n-1)}+\mu_{n-2} p_{2(n-2, n)}=0 ; \\
& -\left(f_{\lambda}(2)+\mu_{i}+\mu_{n}\right) p_{2(i n)}+\mu_{i-1} p_{2(i-1, n)}+\mu_{n-1} p_{2(i, n-1)}=0, \quad 2 \leq i \leq n-2 ; \\
& -\left(f_{\lambda}(k)+2 \mu_{n}\right) p_{k(n n)}+f_{\lambda}(k-1) p_{k-1(n n)}+\mu_{n-1} p_{k(n-1, n)}=0, \quad 3 \leq k \leq m-1 ; \\
& -2 \mu_{n} p_{m(n n)}+f_{\lambda}(m-1) p_{m-1(n n)}+\mu_{n-1} p_{m(n-1, n)}=0 ; \\
& -\left(f_{\lambda}(k)+\mu_{n-1}+\mu_{n}\right) p_{k(n-1, n)}+f_{\lambda}(k-1) p_{k-1(n-1, n)}+2 \mu_{n-1} p_{k(n-1, n-1)}+ \\
& \quad+\mu_{n-2} p_{k(n-2, n)}=0, \quad 3 \leq k \leq m-1 ; \\
& -\left(\mu_{n-1}+\mu_{n}\right) p_{m(n-1, n)}+f_{\lambda}(m-1) p_{m-1(n-1, n)}+2 \mu_{n-1} p_{m(n-1, n-1)}+ \\
& \quad+\mu_{n-2} p_{m(n-2, n)}=0 ; \\
& -\left(f_{\lambda}(k)+\mu_{i}+\mu_{n}\right) p_{k(i n)}+f_{\lambda}(k-1) p_{k-1(i n)}+\mu_{i-1} p_{k(i-1, n)}+ \\
& +\mu_{n-1} p_{k(i, n-1)}=0, \quad 3 \leq k \leq m-1,2 \leq i \leq n-2 ; \\
& -\left(\mu_{i}+\mu_{s}\right) p_{m+2(i s)}+f_{\lambda}(m+1) p_{m+1(i s)}+\mu_{i-1} p_{m+2(i-1, s)}+ \\
& \quad+\mu_{s-1} p_{m+2(i, s-1)}=0, \quad 2 \leq i \leq s-2 .
\end{aligned}
$$

Equations (16) as well as the equation

$$
-\left(f_{\lambda}(1)+\mu_{n}\right) p_{1(0 n)}+2 \mu_{n} p_{2(n n)}+\mu_{n-1} p_{1(0, n-1)}=0,
$$

were not involved in obtaining (15). Using the normalization condition (14), we find the steady-state probabilities by (8).

\section{Numerical example}

Consider a two-channel closed queueing system with an exponential distribution of the time generation of service requests and Erlangian service times of the order $n=10$ for the following values of the parameters: $m=12 ; \lambda=1 ; \mu_{i}=20$, $1 \leq i \leq 10$.

The values of the steady-state characteristics of the system, found using the recurrence relations obtained in this paper, are presented in Tables 1 and 2 . In order to verify the obtained values, the tables contain the computing results obtained with the help of the GPSS World simulation system [6] for the time value $t=10^{6}$. 
Table 1

Stationary distribution of the number of customers in the system

\begin{tabular}{|c|c|c|}
\hline \multirow{2}{*}{$k$} & \multicolumn{2}{|c|}{ Values of the steady-state probabilities $p_{k}$} \\
\cline { 2 - 3 } & Recurrence method & GPSS World \\
\hline 0 & 0.000004 & 0.000004 \\
\hline 1 & 0.000055 & 0.000053 \\
\hline 2 & 0.000440 & 0.000461 \\
\hline 3 & 0.002605 & 0.002620 \\
\hline 4 & 0.011830 & 0.011963 \\
\hline 5 & 0.040833 & 0.040495 \\
\hline 6 & 0.105048 & 0.104882 \\
\hline 7 & 0.196206 & 0.196322 \\
\hline 8 & 0.256876 & 0.256441 \\
\hline 9 & 0.224423 & 0.224365 \\
\hline 10 & 0.121478 & 0.122119 \\
\hline 11 & 0.035863 & 0.036016 \\
\hline 12 & 0.004340 & 0.004259 \\
\hline
\end{tabular}

Table 2

Stationary characteristics of the system

\begin{tabular}{|l|c|c|c|c|}
\hline \multicolumn{1}{|c|}{ Method } & $\mathbf{E}\left(n_{c}\right)$ & $\mathbf{E}(Q)$ & $\mathbf{E}(W)$ & $\lambda_{\mathrm{av}}$ \\
\hline Recurrence & 8.000127 & 6.000191 & 1.500095 & 3.999873 \\
\hline GPSS World & 8.002 & 6.001 & 1.500 & - \\
\hline
\end{tabular}

\section{Conclusions}

The numerical algorithm for solving a system of equations for steady-state probabilities, developed in this article, is based on the presence of three or four unknowns in most equations. The constructed recurrence relations are used for the direct calculation of the steady-state probabilities, that allows us to reduce the amount of calculations in comparison with the case of application of the direct or iterative classical methods. Using the obtained recurrence relations makes it possible in the case of $n=2$ to directly calculate the steady-state probabilities, in the case of $n=3$ to reduce the system of equations for the steady-state probabilities for a single linear equation for the unknown parameter $p$, and in the case of $n \geq 4$ to reduce the number of solved equations from $(n+1)(2+n(m-1)) / 2$ to $n(m-1)$. 


\section{References}

[1] Nesterov, Yu.G. (2014). Analysis of characteristics of a closed queuing system with relative priorities. Nauka i Obrazovanie. MGTU im. N. Baumana, 3, 242-254 (in Russian).

[2] Bocharov, P.P., \& Pechinkin, A.V. (1995). Queueing Theory. Moskow: RUDN (in Russian).

[3] Brockmeyer, E., Halstrøm, H.L., \& Jensen, A. (1948). The Life and Works of A.K. Erlang. Copenhagen: Danish Academy of Technical Sciences.

[4] Zhernovyi, K.Yu. (2017). Determining stationary characteristics of two-channel queueing systems with Erlangian distribution of service time. Cybernetics and Systems Analysis, 53, 1, 92-104.

[5] Kopytko, B., \& Zhernovyi, K. (2016). Steady-state characteristics of three-channel queueing systems with Erlangian service times. Journal of Applied Mathematics and Computational Mechanics, 15(3), 75-87.

[6] Zhernovyi, Yu. (2015). Creating Models of Queueing Systems Using GPSS World. Saarbrücken: LAP Lambert Academic Publishing. 